\title{
A Note on the Characterization of Two-Dimensional Quasi-Einstein Manifolds
}

\author{
Gabriel Bercu \\ Department of Mathematics and Computer Sciences, "Dunărea de Jos" University of Galaţi, \\ 800008 Galaţi, Romania; gbercu@ugal.ro
}

Received: 26 September 2020; Accepted: 10 November 2020; Published: 12 November 2020

\begin{abstract}
In this article, we aim to introduce new classes of two-dimensional quasi-Einstein pseudo-Riemannian manifolds with constant curvature. We also give a classification of $2 D$ quasi-Einstein manifolds of warped product type working in local coordinates. All the results are obtained by elementary methods.
\end{abstract}

Keywords: Ricci soliton; quasi-Einstein metric; curvature; potential function

\section{Introduction}

Nonlinear differential equations arise in wide areas of research in pure and applied sciences. In this note, we study some non-linear differential equations whose solutions are quasi-Einstein metrics that appear in mathematical physics [1,2]. In our approach, we will use the techniques of mathematical analysis, more suitable in this case, as in article [3]. The quasi-Einstein metric is a generalization of the Einstein metric, it contains gradient Ricci solitons and it is also closely related to the construction of the warped product Einstein metrics. The study of quasi-Einstein metrics was initiated by Chaki and Maity in [4]. In [5], Chaki and Ghoshal studied some global properties of quasi-Einstein manifolds, while in [6], De and Ghosh gave some examples, proven their existence, and underline some properties. As applications, gravitational instantons are defined to be solutions of the quasi-Einstein equations $[7,8]$.

To set forth our research in this article, we organized our discussion in two parts.

In the first part of this research, we consider a pseudo-Riemannian manifold $(M, \bar{g})$, which has constant sectional curvature $K$, [9]. Given a smooth real valued mapping $F$, defined on $M$ and $\mu$ a real constant, suppose that the tensor having the components $h_{i j}=F_{, i j}-\mu F_{, i} F_{, j}$ is non-degenerate and has constant signature. With these assumptions, we establish a link between the Christoffel symbols $\Gamma_{i j}^{k}$ of $h$ and the Christoffel symbols $\bar{\Gamma}_{i j}^{k}$ of the initial metric $\bar{g}$.

When $\bar{g}$ has constant sectional curvature $K$, it is known that $\bar{R}_{j l}=(n-1) K \bar{g}_{j l}$. The relation which defines the quasi-Einstein pseudo-Riemannian metrics:

$$
\bar{R}_{i j}+F_{, i j}-\mu F_{, i} F_{, j}=\rho \bar{g}_{i j}
$$

can be written as:

$$
F_{, i j}-\mu F_{, i} F_{, j}=[\rho-(n-1) K] \bar{g}_{i j} .
$$

This means that the Christoffel symbols $\Gamma_{i j}^{k}$ of $h$ and the Christoffel symbols $\bar{\Gamma}_{i j}^{k}$ of $\bar{g}$ have to be identical. The equalities:

$$
\Gamma_{i j}^{k}=\bar{\Gamma}_{i j}^{k}, \forall i, j, k=\overline{1, n}
$$

imply necessary conditions such that the initial metric $\bar{g}$, with constant curvature $K$, becomes a quasi-Einstein pseudo-Riemannian metric. 
As application, we shall show that $\mathbb{R}^{2}$, endowed with the pseudo-Riemann metric of Kruskal-type, having null sectional curvature, is a quasi-Einstein manifold.

The second part of this research aims to continue the study of A. Pitea, recently published in $[10,11]$. Following this research, we introduce the explicit form of the sectional curvature under the necessary conditions that a generalized Poincaré metric $\bar{g}(x, y)=\operatorname{diag}(g(y), f(y))$ becomes quasi-Einstein. We show that if the sectional curvature $K$ is constant, then the metric $\bar{g}$ becomes a quasi-Einstein one.

As application, we consider the warped product Riemannian metrics $\bar{g}(x, y)=\operatorname{diag}(g(y), 1)$, with constant sectional curvature, and we obtain a complete classification of those metrics which are quasi-Einstein too.

\section{A New Class of Quasi-Einstein Metrics}

Finding quasi-Einstein metrics classes has attracted the attention of much research, since there were studied in different forms in the recent past [2,7,10-13].

Consider a smooth $n$-dimensional pseudo-Riemannian manifold $\left(M^{n}, \bar{g}\right)$ and fix $F: M \rightarrow \mathbb{R}$ a smooth real valued function on $M$. One denotes with $\nabla^{2} F$ its Hessian with respect to $\bar{g}$.

A natural extension of the Ricci tensor $\overline{R i c}$ of $\bar{g}$ is the $\mu$-Bakry-Emery Ricci tensor:

$$
\overline{\operatorname{Ric}}_{F}^{\mu}:=\overline{\operatorname{Ric}}+\nabla^{2} F-\mu d F \otimes d F
$$

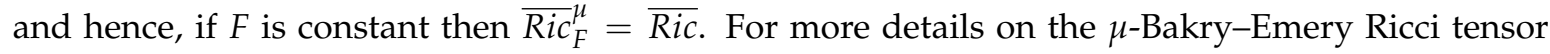
see $[14,15]$.

The pseudo-Riemannian manifold $(M, \bar{g})$ is called quasi-Einstein if the $\mu$-Bakry-Emery Ricci tensor is a constant multiple of the metric tensor:

$$
\overline{\operatorname{Ric}}_{F}^{\mu}=\rho \bar{g}, \rho \in \mathbb{R} .
$$

In local coordinates, the relation (1) becomes a system of differential equations:

$$
\bar{R}_{i j}+F_{, i j}-\mu F_{, i} F_{, j}=\rho \bar{g}_{i j}
$$

for some real constants $\mu$ and $\rho$, where [13]:

$$
F_{, i}=\frac{\partial F}{\partial x^{i}} ; F_{, i j}=\frac{\partial^{2} F}{\partial x^{i} \partial x^{j}}-\bar{\Gamma}_{i j}^{r} F_{, r} ; F_{, i j k}=\frac{\partial F_{, i j}}{\partial x^{k}}-\bar{\Gamma}_{k i}^{l} F_{, l j}-\bar{\Gamma}_{k j}^{l} F_{, l i} .
$$

For $\mu=0$ the relation (1) defines gradient Ricci solitons, [16,17].

Suppose that the tensor of components $h_{i j}=F_{, i j}-\mu F_{, i} F_{, j}$ is non-degenerate and has constant signature. Then $h$ is a new pseudo-Riemannian metric, which has the Levi-Civita connection $\nabla_{h}$ and the Christoffel symbols $\Gamma_{i j}^{k}$, see $[18,19]$. We will derive the symbols $\Gamma_{i j}^{k}$ in terms of the $\bar{\Gamma}_{i j}^{k}$ of $\bar{g}_{i j}$ :

Theorem 1. Let $h^{\rho k}$ be the contravariant components of the pseudo-Riemannian metric $h$ and $\bar{R}_{i j k}^{m}$ the curvature tensor field of $\bar{g}$. Then the Christoffel symbols $\Gamma_{i j}^{p}$ are given by:

$$
\Gamma_{i j}^{p}=\bar{\Gamma}_{i j}^{p}+\frac{1}{2} h^{p k}\left[F_{, i j k}-2 \mu F_{, i j} F_{, k}+\left(\bar{R}_{i k j}^{l}+\bar{R}_{j k i}^{l}\right) F_{, l}\right] .
$$


Proof. From:

$$
\begin{aligned}
\frac{\partial h_{i j}}{\partial x^{k}} & =\frac{\partial}{\partial x^{k}}\left(F_{, i j}-\mu F_{, i} F_{, j}\right) \\
& =\frac{\partial}{\partial x^{k}}\left(\frac{\partial^{2} F}{\partial x^{i} \partial x^{j}}-\bar{\Gamma}_{i j}^{r} F_{, r}\right)-\mu\left(\frac{\partial^{2} F}{\partial x^{k} \partial x^{i}} F_{, j}+F_{, i} \frac{\partial^{2} F}{\partial x^{k} \partial x^{j}}\right) \\
& =\frac{\partial^{3} F}{\partial x^{i} \partial x^{j} \partial x^{k}}-\frac{\partial \bar{\Gamma}_{i j}^{r}}{\partial x^{k}} F_{, r}-\bar{\Gamma}_{i j}^{r} F_{, k r}-\bar{\Gamma}_{i j}^{r} \bar{\Gamma}_{k r}^{l} F_{, l}-\mu\left(\frac{\partial^{2} F}{\partial x^{k} \partial x^{i}} F_{, j}+F_{, i} \frac{\partial^{2} F}{\partial x^{k} \partial x^{j}}\right)
\end{aligned}
$$

and the similar formulas for $\frac{\partial h_{j k}}{\partial x^{i}}$ and $\frac{\partial h_{i k}}{\partial x^{j}}$, we have:

$$
\begin{aligned}
2 h_{p k} \Gamma_{i j}^{p}= & \frac{\partial h_{j k}}{\partial x^{i}}+\frac{\partial h_{i k}}{\partial x^{j}}-\frac{\partial h_{i j}}{\partial x^{k}}=\frac{\partial^{3} F}{\partial x^{i} \partial x^{j} \partial x^{k}}+\left(\frac{\partial \bar{\Gamma}_{i j}^{l}}{\partial x^{k}}-\frac{\partial \bar{\Gamma}_{j k}^{l}}{\partial x^{i}}-\frac{\partial \bar{\Gamma}_{i k}^{l}}{\partial x^{j}}\right) F_{, l}+ \\
& +\left(\bar{\Gamma}_{i j}^{r} \bar{\Gamma}_{k r}^{l}-\bar{\Gamma}_{i k}^{r} \bar{\Gamma}_{j r}^{l}-\bar{\Gamma}_{j k}^{r} \bar{\Gamma}_{i r}^{l}\right) F_{, l}+\left(\bar{\Gamma}_{i j}^{r} F_{, k r}-\bar{\Gamma}_{j k}^{r} F_{, i r}-\bar{\Gamma}_{i k}^{r} F_{, j r}\right)- \\
& -2 \mu \frac{\partial^{2} F}{\partial x^{i} \partial x^{j}} F_{, k} .
\end{aligned}
$$

Substituting $\frac{\partial^{2} F}{\partial x^{i} \partial x^{j}}$ by $F_{, i j}+\bar{\Gamma}_{i j}^{r} F_{, r}$ and using the formula for $F_{, i j k}$ we obtain:

$$
\begin{aligned}
2 h_{p k} \Gamma_{i j}^{p}= & F_{, i j k}+\left(\bar{\Gamma}_{k i}^{l} F_{, l j}+\bar{\Gamma}_{k j}^{l} F_{, l i}+\bar{\Gamma}_{i j}^{l} F_{k l}\right)+\frac{\partial \bar{\Gamma}_{i j}^{l}}{\partial x^{k}} F_{, l}+\bar{\Gamma}_{i j}^{r} \bar{\Gamma}_{k r}^{l} F_{, l}+ \\
& +\left(\frac{\partial \bar{\Gamma}_{i j}^{l}}{\partial x^{k}}-\frac{\partial \bar{\Gamma}_{j k}^{l}}{\partial x^{i}}-\frac{\partial \bar{\Gamma}_{i k}^{l}}{\partial x^{j}}+\bar{\Gamma}_{i j}^{r} \bar{\Gamma}_{k r}^{l}-\bar{\Gamma}_{i k}^{r} \bar{\Gamma}_{j r}^{l}-\bar{\Gamma}_{j k}^{r} \bar{\Gamma}_{i r}^{l}\right) F_{, l}+ \\
& +\left(\bar{\Gamma}_{i j}^{r} F_{, k r}-\bar{\Gamma}_{j k}^{r} F_{, i r}-\bar{\Gamma}_{i k}^{r} F_{, j r}\right)-\left(2 \mu F_{, i j}+2 \mu \bar{\Gamma}_{i j}^{r} F_{, r}\right) F_{, k} .
\end{aligned}
$$

We reduce the terms $\bar{\Gamma}_{k i}^{l} F_{, l j}$ with $\bar{\Gamma}_{i k}^{r} F_{, j r}$ and $\bar{\Gamma}_{k j}^{l} F_{, l i}$ with $-\bar{\Gamma}_{j k}^{r} F_{, i r}$ and we find:

$$
\begin{aligned}
2 h_{p k} \Gamma_{i j}^{p} & =F_{, i j k}-2 \mu F_{, i j} F_{, k}+2 \bar{\Gamma}_{i j}^{l}\left(F_{, k l}-\mu F_{, k} F_{, l}\right)+\left(\bar{R}_{i k j}^{l}+\bar{R}_{j k i}^{l}\right) F_{, l} \\
& =2 \bar{\Gamma}_{i j}^{l} h_{k l}+F_{, i j k}-2 \mu F_{, i j} F_{, k}+\left(\bar{R}_{i k j}^{l}+\bar{R}_{j k i}^{l}\right) F_{, l} .
\end{aligned}
$$

Finally, we obtain the formula:

$$
\Gamma_{i j}^{p}=\bar{\Gamma}_{i j}^{p}+\frac{1}{2} h^{p k}\left[F_{, i j k}-2 \mu F_{, i j} F_{, k}+\left(\bar{R}_{i k j}^{l}+\bar{R}_{j k i}^{l}\right) F_{, l}\right],
$$

and the proof is complete.

In the following, we consider the case when $(M, \bar{g})$ has constant sectional curvature $K$ and then:

$$
\bar{R}_{j k i}^{l}=K\left(\bar{g}_{k}^{l} \bar{g}_{j i}-\bar{g}_{i}^{l} \bar{g}_{j k}\right), \bar{R}_{i j}=\bar{R}_{i l j}^{l}=(n-1) \bar{g}_{i j} .
$$

The relation (2) takes the form:

$$
F_{, i j}-\mu F_{, i} F_{, j}=[\rho-(n-1) K] \bar{g}_{i j} .
$$


From Theorem 1 we get that $\Gamma_{i j}^{p}=\bar{\Gamma}_{i j}^{p}$, for all $i, j, p=\overline{1, n}$ if and only if:

$$
F_{, i j k}-2 \mu F_{, i j} F_{, k}+K\left(2 \bar{g}_{i j} F_{, k}-\bar{g}_{i k} F_{, j}-\bar{g}_{j k} F_{, i}\right)=0, \forall i, j, p=\overline{1, n}
$$

Example 1. We consider an open subset $M$ of $\mathbb{R}^{2}$ endowed with the pseudo-Riemannian metric of Kruskal-type $\bar{g}(x, y)=\left(\begin{array}{cc}0 & \alpha(x) \beta(y) \\ \alpha(x) \beta(y) & 0\end{array}\right)$, where $\alpha, \beta: \mathbb{R} \rightarrow \mathbb{R}^{*}$ are smooth functions. In addition we assume that: $\alpha(x)>0, \beta(y)>0$ and $\mu>0$. From [20] we have $K=0$ and the Christoffel symbols:

$$
\Gamma_{11}^{1}=\frac{\alpha^{\prime}(x)}{\alpha(x)}, \Gamma_{22}^{2}=\frac{\beta^{\prime}(y)}{\beta(y)}, \Gamma_{12}^{1}=\Gamma_{21}^{1}=\Gamma_{12}^{2}=\Gamma_{21}^{2}=\Gamma_{11}^{2}=\Gamma_{22}^{1}=0 .
$$

The system of differential Equation (3) becomes:

$$
\begin{gathered}
\frac{\partial}{\partial x}\left[\frac{\partial^{2} F}{\partial x^{2}}-\frac{\alpha^{\prime}(x)}{\alpha(x)} \frac{\partial F}{\partial x}\right]-2 \frac{\alpha^{\prime}(x)}{\alpha(x)}\left[\frac{\partial^{2} F}{\partial x^{2}}-\frac{\alpha^{\prime}(x)}{\alpha(x)} \frac{\partial F}{\partial x}\right]=2 \mu\left[\frac{\partial^{2} F}{\partial x^{2}}-\frac{\alpha^{\prime}(x)}{\alpha(x)} \frac{\partial F}{\partial x}\right] \frac{\partial F}{\partial x} \\
\frac{\partial}{\partial y}\left[\frac{\partial^{2} F}{\partial x^{2}}-\frac{\alpha^{\prime}(x)}{\alpha(x)} \frac{\partial F}{\partial x}\right]=2 \mu\left[\frac{\partial^{2} F}{\partial x^{2}}-\frac{\alpha^{\prime}(x)}{\alpha(x)} \frac{\partial F}{\partial x}\right] \frac{\partial F}{\partial y} \\
\frac{\partial^{3} F}{\partial x^{2} \partial y}-\frac{\alpha^{\prime}(x)}{\alpha(x)} \frac{\partial^{2} F}{\partial x \partial y}=2 \mu \frac{\partial^{2} F}{\partial x \partial y} \frac{\partial F}{\partial x} \\
\frac{\partial^{3} F}{\partial x \partial y^{2}}-\frac{\beta^{\prime}(y)}{\beta(y)} \frac{\partial^{2} F}{\partial x \partial y}=2 \mu \frac{\partial^{2} F}{\partial x \partial y} \frac{\partial F}{\partial y} \\
\frac{\partial}{\partial x}\left[\frac{\partial^{2} F}{\partial y^{2}}-\frac{\beta^{\prime}(y)}{\beta(y)} \frac{\partial F}{\partial y}\right]=2 \mu\left[\frac{\partial^{2} F}{\partial y^{2}}-\frac{\beta^{\prime}(y)}{\beta(y)} \frac{\partial F}{\partial y}\right] \frac{\partial F}{\partial x} \\
\frac{\partial}{\partial y}\left[\frac{\partial^{2} F}{\partial y^{2}}-\frac{\beta^{\prime}(y)}{\beta(y)} \frac{\partial F}{\partial y}\right]-2 \frac{\beta^{\prime}(y)}{\beta(y)}\left[\frac{\partial^{2} F}{\partial y^{2}}-\frac{\beta^{\prime}(y)}{\beta(y)} \frac{\partial F}{\partial y}\right]=2 \mu\left[\frac{\partial^{2} F}{\partial y^{2}}-\frac{\beta^{\prime}(y)}{\beta(y)} \frac{\partial F}{\partial y}\right] \frac{\partial F}{\partial y} .
\end{gathered}
$$

By integrating with respect to $y$ the Equation (5), we obtain

$$
\frac{\partial^{2} F}{\partial x^{2}}-\frac{\alpha^{\prime}(x)}{\alpha(x)} \frac{\partial F}{\partial x}=e^{2 \mu F} e^{a(x)}
$$

where $a$ is a function which depends on $x$ only. Substituting in Equation (4) and then integrating with respect to $x$, we get $\ln \left[e^{a(x)} e^{2 \mu F}\right]-2 \ln \alpha(x)=2 \mu F+b(y)$, where $b$ is a function, which depends on $y$ only. The last equation leads us to $e^{a(x)}=\alpha^{2}(x) e^{b(y)}$, hence $\frac{e^{a(x)}}{\alpha^{2}(x)}=e^{b(y)}$ must be a positive constant. Then $a(x)=c+2 \ln \alpha(x)$, and $b(y)=c$, so

$$
\frac{\partial^{2} F}{\partial x^{2}}-\frac{\alpha^{\prime}(x)}{\alpha(x)} \frac{\partial F}{\partial x}=e^{2 \mu F} e^{c} \alpha^{2}(x)
$$

In a similar manner, from the last two equations we obtain

$$
\frac{\partial^{2} F}{\partial y^{2}}-\frac{\beta^{\prime}(x)}{\beta(x)} \frac{\partial F}{\partial y}=e^{2 \mu F} e^{k} \beta^{2}(y)
$$

From the Equation (6) we have $\frac{\partial^{2} F}{\partial x \partial y}=\alpha(x) e^{2 \mu F} e^{p(y)}$ and from the Equation (7) we deduce $\frac{\partial^{2} F}{\partial x \partial y}=$ $\beta(x) e^{2 \mu F} e^{s(x)}$. The last equalities lead us to $\frac{e^{s(x)}}{\alpha(x)}=\frac{e^{p(y)}}{\beta(y)}$, so these expressions must be a positive constant $r$. 
Therefore $e^{s(x)}=r \alpha(x), e^{p(y)}=r \beta(y)$ and $\frac{\partial^{2} F}{\partial x \partial y}=r \alpha(x) \beta(y) e^{2 \mu F}$. Turning back to the initial system (2) which defines quasi-Einstein metrics, we have

$$
\begin{aligned}
& F_{, 11}-\mu F_{, 1} F_{, 1}=\rho \bar{g}_{11}, \\
& F_{, 12}-\mu F_{, 1} F_{, 2}=\rho \bar{g}_{12}, \\
& F_{, 22}-\mu F_{, 2} F_{, 2}=\rho \bar{g}_{22} .
\end{aligned}
$$

The Equation (10) leads us to

$$
\frac{\partial F}{\partial x}= \pm \frac{1}{\sqrt{\mu}} e^{\frac{c}{2}} e^{\mu F} \alpha(x) .
$$

The Equation (12) is equivalent to

$$
\frac{\partial F}{\partial y}= \pm \frac{1}{\sqrt{\mu}} e^{\frac{k}{2}} e^{\mu F} \beta(x) .
$$

The Equation (11)

$$
r \alpha(x) \beta(y) e^{2 \mu F}-\mu \frac{\partial F}{\partial x} \frac{\partial F}{\partial y}=\rho \alpha(x) \beta(y)
$$

becomes $e^{2 \mu F}\left(r \pm e^{\frac{c}{2}} e^{\frac{k}{2}}\right)=\rho$. If $r \pm e^{\frac{c}{2}} e^{\frac{k}{2}} \neq 0$, then $F$ is a constant, a trivial case. So we have to impose $r-e^{\frac{c}{2}} e^{\frac{k}{2}}=0$ and $\rho=0$. Then we choose $\frac{\partial F}{\partial x}=-\frac{1}{\sqrt{\mu}} e^{\mu F} e^{\frac{k}{2}} \alpha(x)$ and $\frac{\partial F}{\partial y}=-\frac{1}{\sqrt{\mu}} e^{\mu F} e^{\frac{k}{2}} \beta(y)$. The Equation (10) becomes

$$
\frac{\partial F}{\partial x} e^{-\mu F}=-\frac{1}{\sqrt{\mu}} e^{\frac{c}{2}} \alpha(x)
$$

hence

$$
e^{-\mu F}=e^{\frac{c}{2}} \sqrt{\mu} \int \alpha(x) d x+t(y) .
$$

The relation (11) can be written

$$
\frac{\partial F}{\partial y} e^{-\mu F}=-\frac{1}{\sqrt{\mu}} e^{\frac{k}{2}} \beta(y)
$$

hence

$$
e^{-\mu F}=e^{\frac{k}{2}} \sqrt{\mu} \int \beta(x) d x+u(x) .
$$

These last two equations lead us to

$$
e^{\frac{c}{2}} \sqrt{\mu} \int \alpha(x) d x-u(x)=e^{\frac{k}{2}} \sqrt{\mu} \int \beta(y) d y-t(y) .
$$

Since the left-hand side is a function with one only variable $x$, and the right-hand side is a function with one only variable $y$, then

$$
\left\{\begin{array}{l}
e^{\frac{c}{2}} \sqrt{\mu} \int \alpha(x) d x-u(x)=-l \\
e^{\frac{k}{2}} \sqrt{\mu} \int \beta(y) d y-t(y)=-l
\end{array}\right.
$$

1 real constant. Thus

$$
u(x)=e^{\frac{c}{2}} \sqrt{\mu} \int \alpha(x) d x+l, t(y)=e^{\frac{k}{2}} \sqrt{\mu} \int \beta(y) d y+l
$$

and

$$
e^{-\mu F}=\sqrt{\mu}\left(e^{\frac{c}{2}} \int \alpha(x) d x+e^{\frac{k}{2}} \int \beta(y) d y\right)+l .
$$


Finally, the potential function is

$$
F(x, y)=\frac{-1}{\mu} \ln \left|\sqrt{\mu}\left(e^{\frac{c}{2}} \int \alpha(x) d x+e^{\frac{k}{2}} \int \beta(y) d y\right)+l\right|,
$$

which corresponds to $\mu \neq 0$ and $\rho=0$.

\section{Generalizing a Question of Besse}

An important question was stated by Besse [21], which was how to determine examples of Einstein manifolds, which are warped products [16]. A natural generalization asks to finding examples of quasi-Einstein manifolds of warped product type. In the following, we continue the study started by Pitea [10], by finding new classes of explicit quasi-Einstein Riemannian manifolds, endowed with generalized Poincaré metrics, which also have constant sectional curvature. We consider the manifold $M$ as an open subset of $\mathbb{R}^{2}$, endowed with metric of diagonal type $\bar{g}(x, y)=\operatorname{diag}(g(y), f(y))$, where $g$ and $f$ are strictly positive smooth functions $[16,22,23]$. In order to find $g, f$ and $F$ which satisfy (2), Pitea [10] shows that in the case when the potential function $F$ depends only on $y$, $F$ has the form:

$$
F(y)=2 \rho \int \frac{f(y) g(y)}{g^{\prime}(y)} d y+\ln \left(\frac{\left|g^{\prime}(y)\right|}{\sqrt{f(y) g(y)}}\right)
$$

Also, Pitea [10] proves that if we introduce new functions $h=\frac{g^{\prime}}{g}, p=\frac{f^{\prime}}{f}$, then from (2) $h$ and $p$ must satisfy the equation:

$$
2 \rho\left(\frac{f}{h}\right)^{\prime}+\left(\frac{h^{\prime}}{h}\right)^{\prime}-\frac{1}{2} p^{\prime}-\mu\left(2 \rho \frac{f}{h}+\frac{h^{\prime}}{h}+\frac{1}{2} h-\frac{1}{2} \rho\right)^{2}=\rho f+\frac{1}{4} h^{2}+\rho p f \frac{1}{h}+\frac{1}{2} p \frac{h^{\prime}}{h}-\frac{1}{4} p^{2} .
$$

Now, we introduce the expression of the sectional curvature:

$$
K=\frac{1}{f(y)}\left[-\left(\frac{g^{\prime}(y)}{2 g(y)}\right)^{\prime}+\frac{g^{\prime}(y)}{2 g(y)}\left(\frac{f^{\prime}(y)}{2 f(y)}-\frac{g^{\prime}(y)}{2 g(y)}\right)\right]
$$

in the Equation (13). It follows $K=\frac{h}{2 f}\left(-\frac{h^{\prime}}{h}+\frac{p-h}{2}\right)$, hence

$$
\frac{h^{\prime}}{h}=\frac{p-h}{2}-2 K \frac{f}{h}
$$

Substituting in (13) we obtain:

$$
\begin{gathered}
\left(\frac{2 \rho f}{h}+\frac{p-h}{2}-\frac{2 K f}{h}\right)^{\prime}-\frac{1}{2} p^{\prime}-\mu\left(\frac{2 \rho f}{h}+\frac{p-h}{2}-\frac{2 K f}{h}+\frac{h-p}{2}\right)^{2} \\
=\rho f+\frac{1}{4} h^{2}+\rho p f \frac{1}{h}+\frac{p}{2}\left(\frac{p-h}{2}-2 K \frac{f}{h}\right)-\frac{1}{4} p^{2}
\end{gathered}
$$

which is equivalent to:

$$
\left(\frac{2 f(\rho-K)}{h}\right)^{\prime}-\frac{1}{2} h^{\prime}-\mu \frac{4 f^{2}(\rho-K)^{2}}{h^{2}}=\rho f+\frac{1}{4} h^{2}+\frac{\rho p f-K p f}{h}-\frac{p h}{4}
$$


Substituting again $h^{\prime}$ by $\frac{h(p-h)}{2}-2 K f$ we find:

$$
2(\rho-K)\left(\frac{f}{h}\right)^{\prime}-4 \mu(\rho-K)^{2} \frac{f^{2}}{h^{2}}=(\rho-K) f+\frac{p f(\rho-K)}{h}
$$

or:

$$
(\rho-K)\left[2 \frac{f^{\prime}}{h}-\frac{2 f}{h} \frac{h^{\prime}}{h}-4 \mu(\rho-K)\left(\frac{f}{h}\right)^{2}-f-\frac{p f}{h}\right]=0 .
$$

But $\frac{h^{\prime}}{h}=\frac{p-h}{2}-2 K \frac{f}{h}$ and $p f=f^{\prime}$. Finally, we get:

$$
(\rho-K)(K-\mu \rho+\mu K)=0 .
$$

The sectional curvature $K$ must be either $K=\rho$ or $K=\frac{\mu \rho}{1+\mu}, \mu \neq-1$. Therefore the use of $K$ from (14) seems be the most suitable way to find quasi-Einstein manifolds with constant sectional curvature, endowed with generalized Poincaré metrics.

Hence we expressed the relation (13) according to sectional curvature $K$, obtaining the relation (14) and the following theorem.

Theorem 2. An open subset $M$ of $\mathbb{R}^{2}$ with the metric $\bar{g}(x, y)=\operatorname{diag}(g(y), f(y))$, having the constant sectional curvature $K=\rho$ or $K=\frac{\mu \rho}{1+\mu}, \mu \neq-1$ is a quasi-Einstein Riemannian manifold corresponding to the real constants $\rho$ and $\mu$.

CASE STUDY. In the following, we shall determine all metrics of the form $\bar{g}(x, y)=$ $\operatorname{diag}(g(y), 1)$ with constant sectional curvature. Taking $f(y)=1$ in the above formula we obtain the sectional curvature:

$$
K=-\frac{1}{2}\left(\frac{g^{\prime}(y)}{g(y)}\right)^{\prime}-\frac{1}{4}\left(\frac{g^{\prime}(y)}{g(y)}\right)^{2},
$$

and the expression of potential function:

$$
F(y)=2 \rho \int \frac{g(y)}{g^{\prime}(y)} d y+\ln \left(\left|\frac{g^{\prime}(y)}{\sqrt{g(y)}}\right|\right) .
$$

The relation $h=\frac{g^{\prime}}{g}$ yields:

$$
2 h^{\prime}=-h^{2}-4 K
$$

The solutions of Equation (15), obtained in the general case, enable us to classify the two dimensional quasi-Einstein Riemannian metrics $\bar{g}(x, y)=\operatorname{diag}(g(y), 1)$ with constant sectional curvature $K$.

CLASs 1. If $-h^{2}-4 K=0$ then $h^{\prime}=0$. Hence $h(y)=c$ a real constant, the metric component $g(y)=e^{c y}$ and the potential function is $F(y)=\frac{4 \rho+c^{2}}{2 c} y+\ln |c|$. If $K=-\rho$ then $\rho=-\frac{c^{2}}{4}$ and $F(y)=\ln |c|$ is trivial. If $K=\frac{\mu \rho}{1+\mu}=-\frac{c^{2}}{4}$ then $F(y)=-\frac{c^{2}}{4} y+\ln |c|$.

Therefore, we just proved the following proposition. 
Proposition 1. The Riemannian manifold $\left(\mathbb{R}^{2}, \bar{g}(x, y)=\operatorname{diag}\left(e^{c y}, 1\right)\right)$ with sectional curvature $K=-\frac{c^{2}}{4}$ is a quasi-Einstein manifold corresponding to the real constants $\rho$ and $\mu$, with $\frac{\mu \rho}{1+\mu}=-\frac{c^{2}}{4}<0, \mu \notin\{-1,0\}$ and the potential function:

$$
F(y)=\frac{-c}{2 \mu} y+\ln |c|
$$

CLASS 2. If $-h^{2}-4 K \neq 0$ then we may write $\frac{2 h^{\prime}}{-h^{2}-4 K}=1$ or $\frac{h^{\prime}}{h^{2}+4 K}=-\frac{1}{2}$.

CLASS 2.1. If $K>0$, then we integrate $\frac{h^{\prime}(y)}{h^{2}(y)+(2 \sqrt{K})^{2}}=-\frac{1}{2}$ and we have $h(y)=$ $-2 \sqrt{K} \tan (y \sqrt{K}), g(y)=\cos ^{2} y \sqrt{K}$ and the potential function

$$
F(y)=\left(\frac{-\rho}{K}+1\right) \ln |\sin y \sqrt{K}|+\ln (2 \sqrt{K}) .
$$

If $K=\rho$ then $F(y)=\ln (2 \sqrt{K})$ is trivial. If $K=\frac{\mu \rho}{1+\mu}$, then $F(y)=-\frac{1}{\mu} \ln |\sin y \sqrt{K}|+\ln (2 \sqrt{K})$.

Therefore, we just proved the following proposition.

Proposition 2. The Riemannian manifold $\left(\mathbb{R} \times\left[-\frac{\pi}{2 K}, \frac{\pi}{2 K}\right], \bar{g}(x, y)=\operatorname{diag}\left(\cos ^{2} y \sqrt{K}, 1\right)\right)$ with the sectional curvature $K>0$ is a quasi-Einstein manifold corresponding to the real constants $\rho$ and $\mu$ with $\frac{\mu \rho}{1+\mu}=K>0, \mu \notin\{-1,0\}$ and the potential function:

$$
F(y)=\frac{-1}{\mu} \ln |\sin y \sqrt{K}|+\ln (2 \sqrt{K})
$$

CLASS 2.2. If $K=0$ then $\frac{h^{\prime}}{h^{2}}=-\frac{1}{2}$ hence $h(y)=-\frac{2}{y}, g(y)=c y^{2}, c>0$ and the potential function:

$$
F(y)=\frac{\rho}{2} y^{2}+\ln 2 \sqrt{c}
$$

If $K=\rho=0$ then $F(y)=\ln 2 \sqrt{c}$ is trivial. If $K=\frac{\mu \rho}{1+\mu}=0$ then we choose $\mu=0$ and in this case we obtain a gradient Ricci soliton.

Hence we just demonstrated the following proposition.

Proposition 3. The Riemannian manifold $\left(\mathbb{R}^{2} \backslash\{0\}, \bar{g}_{c}(x, y)=\operatorname{diag}\left(c y^{2}, 1\right)\right), c>0$ with the sectional curvature $K=0$, is quasi-Einstein corresponding to the real constants $\rho$ and $\mu=0$ and with the potential function:

$$
F(y)=\frac{\rho}{2} y^{2}+\ln 2 \sqrt{c} .
$$

In fact, $\left(\mathbb{R}^{2} \backslash\{0\}, \bar{g}_{c}\right)$ is a gradient Ricci soliton; more precisely, $\bar{g}_{1}$ is the Euclidean metric in polar coordinates.

CLASS 2.3. If $K<0$ then we have $\frac{h^{\prime}}{h^{2}-(2 \sqrt{-K})^{2}}=-\frac{1}{2}$, hence $\left|\frac{h(y)-2 \sqrt{-K}}{h(y)+2 \sqrt{-K}}\right|=e^{-2 \sqrt{-K} y}$ or $\frac{h(y)-2 \sqrt{-K}}{h(y)+2 \sqrt{-K}}= \pm e^{-2 \sqrt{-K} y}$. 
SUBCLASS 2.3.1. If $\frac{h(y)-2 \sqrt{-K}}{h(y)+2 \sqrt{-K}}=e^{-2 \sqrt{-K} y}$ then:

$$
\begin{gathered}
h(y)=2 \sqrt{K} \frac{e^{2 \sqrt{-K} y}}{e^{2 \sqrt{-K} y}}=2 \sqrt{-K} \frac{\operatorname{ch}(\sqrt{-K} y)}{\operatorname{sh}(\sqrt{-K} y)}, \\
g(y)=\operatorname{csh}^{2}(\sqrt{-K} y), \quad c>0
\end{gathered}
$$

and the potential function:

$$
F(y)=\left(-\frac{\rho}{K}+1\right) \ln |\operatorname{ch} \sqrt{-K} y|+\ln (2 \sqrt{-c K}) .
$$

If $K=\rho=0$ then $F(y)=\ln (2 \sqrt{-c K})$ is trivial. If $K=\frac{\mu \rho}{1+\mu}<0$ then $F(y)=-\frac{1}{\mu} \ln |\operatorname{ch} \sqrt{-K y}|+$ $\ln (2 \sqrt{-c K})$.

We just showed the following proposition.

Proposition 4. The Riemannian manifold $\left(\mathbb{R}^{2}, \bar{g}(x, y)=\operatorname{diag}\left(c \operatorname{sh}^{2}(\sqrt{-K} y), 1\right)\right), c>0$ with sectional curvature $K<0$ is a quasi-Einstein corresponding to the real constants $\rho$ and $\mu$ with $\frac{\mu \rho}{1+\mu}=K, \mu \notin\{-1,0\}$ and the potential function:

$$
F(y)=-\frac{1}{\mu} \ln |\operatorname{ch} \sqrt{-K} y|+\ln (2 \sqrt{-c K}) .
$$

SubCLASS 2.3.2. If $\frac{h(y)-2 \sqrt{-K}}{h(y)+2 \sqrt{-K}}=-e^{-2 \sqrt{-K} y}$, then:

$$
h(y)=2 \sqrt{-K} \frac{\operatorname{sh}(\sqrt{-K} y)}{\operatorname{ch}(\sqrt{-K} y)}, \quad g(y)=c \operatorname{ch}^{2}(\sqrt{-K} y), \quad c>0
$$

and the potential function:

$$
F(y)=\left(-\frac{\rho}{K}+1\right) \ln |\operatorname{sh} \sqrt{-K} y|+\ln (2 \sqrt{-c K}) .
$$

If $K=\rho<0$, then $F(y)=\ln (2 \sqrt{-c K})$ is trivial. If $K=\frac{\mu \rho}{1+\mu}<0$ then $F(y)=-\frac{1}{\mu} \ln |\operatorname{sh} \sqrt{-K y}|+$ $\ln (2 \sqrt{-c K})$.

Thus, we obtain the following result.

Proposition 5. The Riemannian manifold $\left(\mathbb{R}[0, \infty), \bar{g}(x, y)=\operatorname{diag}\left(c \operatorname{ch}^{2}(\sqrt{-K} y), 1\right)\right), c>0$ with the sectional curvature $K<0$ is quasi-Einstein corresponding to the real constants $\rho$ and $\mu$, with $\frac{\mu \rho}{1+\mu}=K, \mu \notin$ $\{-1,0\}$ and the potential function:

$$
F(y)=-\frac{1}{\mu} \ln |\operatorname{sh} \sqrt{-K} y|+\ln (2 \sqrt{-c K}) .
$$

Remark 1. The pseudo-Riemannian metric $\bar{g}(x, y)=\operatorname{diag}\left(c \operatorname{ch}^{2}(\sqrt{-K} y), 1\right)$ corresponding to $c=-1$ is exactly the two-dimensional version of anti de Sitter metric of General Relativity, a very important model in that theory. 


\section{Conclusions}

In our work, we provided new classes of two-dimensional quasi-Einstein manifolds, endowed with generalized Poincaré metrics and having constant sectional curvature $K$.

These metrics generalize some remarkable metrics (for instance, Kruskal-type metric and anti de Sitter metric) and have applications in Theoretical and Experimental Physics [24-30], where geometric methods are used for physical modeling.

This use is due to the fact that the Ricci tensor for these manifolds looks like that of a perfect fluid model of general relativity.

Funding: The APC was funded by “Dunărea de Jos” University of Galaţi, Romania.

Conflicts of Interest: The author declares that he has no competing interests.

Availability of Data and Material: The datasets used or analyzed during the current study are available from the corresponding author on reasonable request.

\section{References}

1. He, C.; Petersen, P.; Wylie, W. On the classification of warped product Einstein metrics. Commun. Anal. Geom. 2012, 20, 271-311. [CrossRef]

2. Friedan, D. Nonlinear models in $2+\varepsilon$ dimensions. Ann. Phys. 1985, 163, 318-419. [CrossRef]

3. Eminenti, M.; Nave, G.L.; Mantegazza, C. Ricci solitons: The equation point of view. Man. Math. 2008, 127, 345-367. [CrossRef]

4. Chaki, M.C.; Maity, R.K. On quasi-Einstein manifolds. Publ. Math. 2000, 57, 297-306.

5. Chaki, M.C.; Ghoshal, P.K. Some global properties on quasi-Einstein manifolds. Publ. Math. 2003, 63, 635-641.

6. De, U.C.; Ghosh, G.C. On quasi-Einstein manifolds. Period. Math. Hung. 2004, 48, 223-231. [CrossRef]

7. Hall, S.J. Quasi-Einstein metrics on hypersurface families. J. Geom. Phys. 2013, 64, 83-90. [CrossRef]

8. Page, D. A compact rotating gravitational instanton. Phys. Lett. B 1978, 79, 235-238. [CrossRef]

9. Bercu, G.; Corcodel, C.; Postolache, M. On a study of distinguished structures of Hessian type on pseudo-Riemannian manifolds. J. Adv. Math. Stud. 2009, 2, 1-16.

10. Pitea, A. On new classes of explicit quasi-Einstein Riemannian manifolds. Int. J. Geom. Methods Mod. Phys. 2012, 9, 1220015. [CrossRef]

11. Pitea, A. A geometric study of some equations of Mathematical Physics. Int. J. Geom. Methods Mod. Phys. 2012, 9, 1250030. [CrossRef]

12. Duistermaat, J. On Hessian Riemannian structures. Asian J. Math. 2001, 5, 79-91. [CrossRef]

13. Case, J.; Shu, Y.-J.; Wei, G. Rigidity of quasi-Einstein metrics. Diff. Geometry Appl. 2011, 29, 93-100. [CrossRef]

14. Fasili-Ramandi, G. The Bakry-Emery Ricci tensor: Application to mass distribution in space-time. arXiv 2019, arXiv:1902.04428v1[math.GM].

15. Wei, G.; Wylie, W. Comparison geometry for the Bakry-Emery Ricci tensor. J. Diff. Geom. 2009, 83, 377-405. [CrossRef]

16. Bercu, G.; Postolache, M. Classes of gradient Ricci solitons on generalized Poincaré manifolds. Int. J. Geom. Methods Mod. Phys. 2012, 9, 16. [CrossRef]

17. Bercu, G.; Postolache, M. Classification of steady gradient Ricci solitons on two-manifolds. Int. J. Geom. Methods Mod. Phys. 2012, 9, 9. [CrossRef]

18. Shima, H. The Geometry of Hessian Structures; World Scientific Publ. Co.: Singapore, 2007.

19. Udrişte, C.; Bercu, G.; Postolache, M. 2D Hessian Riemannian manifolds. J. Adv. Math. Stud. 2008, 1, 135-142.

20. Bercu, G. 2D Ricci flat gradient solitons arising from remarkable models in Physics. Int. J. Geom. Methods Mod. Phys. 2013, 10, 12. [CrossRef]

21. Besse, A.L. Einstein Manifolds; Springer: Berlin/Heidelberg, Germany; New York, NY, USA, 1987.

22. Barros, A.; Ribeiro, E., Jr. Integral formulae on quasi-Einstein manifolds and applications. Glasg. Math. J. 2012, 54, 213-223. [CrossRef]

23. Bercu, G.; Corcodel, C.; Postolache, M. Iterative geometric structures. Int. J. Geom. Methods Mod. Phys. 2010, 7, 1103-1114. [CrossRef] 
24. Cao, H.D. Geometry of Ricci solitions. Chin. Ann. Math. Ser. B 2006, 27, 121-142. [CrossRef]

25. Fernández-Lopez, M.; Garcia-Rfio, E. A remark on compact Ricci solitons. Math. Ann. 2008, 340, $893-896$. [CrossRef]

26. Guan, D.Z.-D. Quasi-Einstein metrics. Interat. J. Math. 1995, 6, 371-379. [CrossRef]

27. Koiso, N. On Rotationally symmetric Hamilton's equations for Kähler-Einstein metrics. Adv. Stud. Pure Math. 1990, 18, 327-337.

28. Lee, H.K. The fundamental groups of $m$-quasi-Einstein manifolds. ISRN Geom. 2011, 812541. [CrossRef]

29. Marin, M. On the minimum principle for dipolar materials with stretch. Nonlinear Anal. Real World Appl. 2009, 10, 1572-1578. [CrossRef]

30. Filho, J.F.S. Quasi-Einstein manifolds endowed with a parallel vector field. Monatsh. Math. 2016, 179, 305-320. [CrossRef]

Publisher's Note: MDPI stays neutral with regard to jurisdictional claims in published maps and institutional affiliations.

(C) 2020 by the author. Licensee MDPI, Basel, Switzerland. This article is an open access article distributed under the terms and conditions of the Creative Commons Attribution (CC BY) license (http:// creativecommons.org/licenses/by/4.0/). 